\title{
Band Alignment Engineers Faradaic and Capacitive Photostimulation of Neurons Without Surface Modification
}

\author{
Shashi Bhushan Srivastava, ${ }^{1}$ Rustamzhon Melikov, ${ }^{1}$ Mohammad Mohammadi Aria, ${ }^{2}$ Ugur Meric \\ Dikbas, ${ }^{3}$ Ibrahim Halil Kavakli, ${ }^{3,4}$ and Sedat Nizamoglu ${ }^{1,2, *}$ \\ ${ }^{1}$ Department of Electrical and Electronics Engineering, Koc University, Istanbul 34450, Turkey \\ ${ }^{2}$ Graduate School of Biomedical Sciences and Engineering, Koc University, Istanbul 34450, Turkey \\ ${ }^{3}$ Molecular Biology and Genetics, College of Science, Koc University, Istanbul 34450, Turkey \\ ${ }^{4}$ College of Engineering, Chemical and Biological Engineering, Koç University, Istanbul 34450, Turkey
}

(Received 1 November 2018; revised manuscript received 20 February 2019; published 4 April 2019; corrected 17 June 2019)

\begin{abstract}
Photovoltaic substrates have attracted significant attention for neural photostimulation. The control of the Faradaic and capacitive (non-Faradaic) charge transfer mechanisms by these substrates are critical for safe and effective neural photostimulation. We demonstrate that the intermediate layer can directly control the strength of the capacitive and Faradaic processes under physiological conditions. To resolve the Faradaic and capacitive stimulations, we enhance photogenerated charge density levels by incorporating $\mathrm{PbS}$ quantum dots into a poly(3-hexylthiophene-2,5-diyl):([6,6]-Phenyl-C61-butyric acid methyl ester (P3HT:PCBM) blend. This enhancement stems from the simultaneous increase of absorption, well matched band alignment of PbS quantum dots with $\mathrm{P} 3 \mathrm{HT}: \mathrm{PCBM}$, and smaller intermixed phase-separated domains with better homogeneity and roughness of the blend. These improvements lead to the photostimulation of neurons at a low light intensity level of $1 \mathrm{~mW} \mathrm{~cm} \mathrm{~cm}^{-2}$, which is within the retinal irradiance level. These findings open up an alternative approach toward superior neural prosthesis.
\end{abstract}

DOI: 10.1103/PhysRevApplied.11.044012

\section{INTRODUCTION}

Light activation of neurons by planar interfaces is a developing field with applications extending from the fundamental examination of neuronal systems to the development of advanced implants such as artificial retinas $[1,2]$. Advantageously, light can be effectively focused to a spot that enables precise and local perturbation of the discrete parts of a single neuron [3,4]. Moreover, light can be adjusted to remotely control the strength of the transmembrane potential and it also allows for separation of cell stimulation and recording channels [5]. Hence, light stimulation allows spatially and temporally precise excitation and inhibition of neurons both in vitro and in vivo, and can be used for biomedical applications such as drug screening, basic neurological treatments, and light-activated implants [6-8].

For neural photostimulation, photovoltaic substrates have attracted significant attention and the control of the stimulating charge-transfer mechanisms by these substrates is critical for safe and effective cell stimulation. Today, neural implants in the clinics use two major stimulation mechanisms, which are based on Faradaic and capacitive (non-Faradaic) charge-transfer processes

\footnotetext{
*snizamoglu@ku.edu.tr
}

[9-15]. In the Faradaic process, electrons are transferred between the electrode and electrolyte via oxidation and reduction reactions. In the capacitive process, charges electrostatically perturb the ions in the electrode/electrolyte interface and lead stimulating currents $[16,17]$. Just as in clinical electrodes, it is also desirable to control the chargetransfer mechanisms by photovoltaic substrates. So far, optically-active substrates using different conjugated polymer material systems such as poly(3-hexylthiophene-2,5diyl (P3HT), P3HT:[6,6]-Phenyl-C61-butyric acid methyl ester (PCBM), poly(3-octylthiophene): $\operatorname{poly}\left\{\left[\mathrm{N}, \mathrm{N}^{\prime}\right.\right.$-bis $(2-$ octyldodecyl)-naphthalene-1, 4, 5, 8-bis(dicarboximide)-2, 6-diyl]-alt-5,5'-(2,2'-bithiophene)\} (P3OT:N2200), Poly\{2, $2^{\prime}$-[(2,5-bis(2-hexyldecyl)-3,6-dioxo-2,3,5,6- tetrahydropy rrolo[3,4-c]pyrrole-1,4-diyl)dithiophene]- 5,5'-diyl-alt-thio phen-2,5-diyl\} (PDPP3T):PCBM, and P3HT:N2200 have been demonstrated for photostimulation [18-23]. In the previous reports, the control on Faradaic vs capacitive contributions is generally realized by varying the polymeric materials interfacing with cells. Moreover, in some cases, the charge density levels are not high enough to resolve the Faradaic and capacitive contributions [24]. To resolve the contribution by each mechanism, light intensity levels can be increased, but this can lead to thermocapacitive effects on the membrane $[25,26]$, which need to be minimized to clearly observe the charge-transfer mechanisms. 
To address these fundamental challenges, we show a clear control and identification of Faradaic and capacitive contributions on photostimulation at light intensity levels within the retinal irradiance level. For this, we incorporate colloidal quantum dots (QDs) into an organic bulk heterojunction (i.e., photoactive layer) that can strongly absorb the visible spectrum and that is well matched with the band alignment of the donoracceptor polymers. Moreover, in metallic electrodes, the surface material type and porosity are also varied to tune the stimulation strength and mechanism [27-30]. As an unconventional approach, we demonstrate that while the photoactive layer interfacing with the cells is kept fixed, band engineering of the intermediate layer between the photoactive layer and conductive substrate enables tuning of the capacitive and Faradaic stimulation mechanisms. We justify the charge-injection mechanisms with single-cell electrophysiology experiments and numerical simulations using a two-domain stimulation model [31].

\section{METHODS}

\section{A. Photoelectrode fabrication}

The $95.7 \%$ regioregular P3HT, $>99 \%$ pure PCBM, and $\mathrm{PbS}$ QDs in toluene-photosensitive materials are used as received commercially without any modification. The size of the $\mathrm{PbS}$ QDs is $3.7 \mathrm{~nm}$ according to the absorbance data (Appendix A) [32]. It has oleic acid as a ligand [33,34]. The photoactive solution is prepared by mixing two donor materials (P3HT and PbS QDs) and an acceptor (PCBM) material in o-dichlorobenzene solvent. The blending ratio of the PbS in the P3HT:PbS QDs:PCBM blend is 1:0.17:1. The energy levels of each material are calculated from cyclic voltammetry measurements discussed in Appendix $\mathrm{A}$ and are close to the literature values $[32,35,36]$.

To study the effects of various functional interfaces, the ITO electrode and photovoltaic layer are set aside for each photoelectrode. We prepare three types of photoelectrodes by changing the intermediate layer between ITO and a photoactive thin film. The type I (ITO/P3HT:PbS QDs: PCBM) photoelectrode is fabricated by spin coating the photoactive solution onto an ITO electrode at $2000 \mathrm{rpm}$ and annealing at $155^{\circ} \mathrm{C}$ for $15 \mathrm{~min}$. The thickness of the photoactive thin film is found to be $83 \mathrm{~nm}$. The type II (ITO/ $\mathrm{MoO}_{\mathrm{x}} / \mathrm{P} 3 \mathrm{HT}:$ PbSQDs: $\mathrm{PCBM}$ ) photoelectrode is fabricated by using a 10-nm thick $\mathrm{MoO}_{\mathrm{x}}$ layer, processed by thermal evaporation at the rate of $0.03 \mathrm{~nm} / \mathrm{s}$ under a vacuum of $2.0 \times 10^{-6} \mathrm{mbar}$, between the ITO and photoactive layers. Similarly, the type III (ITO/ZnO/P3HT:PbSQDs: PCBM) photoelectrode has an additional $\mathrm{ZnO}$ layer of 49$\mathrm{nm}$ thickness, processed by the sol-gel method, between the ITO and photoactive layers. The thicknesses of various layers are confirmed by SEM cross-section imaging (See Supplemental Material [37] Fig. S1).

\section{B. Electrochemical measurement of photoelectrodes}

To study the interfacial photo-Faradaic and photocapacitive physical phenomena, impedance spectroscopy (IS) measurements are performed on photoelectrodes with different functional interfaces under blue light-emitting diode (LED) light. Using IS, multiple charge carrier accumulation and charge transport can be distinguished at various interfaces in the device according to their response against an externally applied ac signal. Hence, the samples are probed under $20 \mathrm{mV}$ (Vrms) ac perturbation that varies over a frequency range of $1 \mathrm{~Hz}$ to $1 \mathrm{MHz}$ at zero de bias under light conditions (at a wavelength of $450 \mathrm{~nm}$ with an intensity level of $1 \mathrm{~mW} \mathrm{~cm}^{-2}$ ).

For quantitative analysis, the impedance data are modeled using an equivalent electrical circuit with the circuit elements, which are assigned to various physical phenomena in the device. The random characteristics of the blend photoactive layer can be attributed to the distribution of relaxation times and they are represented by the constant phase element (CPE) with impedance $Z=Y_{0}^{-1}(j \omega)^{-N}$, where $Y_{0}$ is the coefficient of the CPE and $N$ represents an ideality factor. $N$ varies from 0 to 1.0 , with 0 corresponding to pure resistive and 1.0 corresponding to pure capacitive behavior [38-40]. The fitted parameters allow us to calculate the capacitance between the photoelectrode/electrolyte (extracellular medium) interface [41].

\section{Optical excitation: photocurrent measurement}

The photostimulation is performed on a set up comprising a Olympus T2 upright microscope and is placed inside a Faraday cage to prevent electrical noise. An extracellular patch clamp (EPC) 800 patch clamp amplifier (HEKA Elektronik) is used for the photocurrent measurements. For the light stimulation, we use a blue LED. A power meter (Newport 843-R) is used to measure the exact power of light reaching the interface. The illumination is focused at a water immersion objective $(40 \mathrm{x} / 0.8 \mathrm{~W}$, inf/0/FN 26.5) from the ITO (a transparent electrode) side of the photoelectrode.

\section{Electrophysiology}

An EPC 800 patch clamp amplifier (HEKA Elektronik) is used for the electrophysiology and the photocurrent measurements. Pulled patch pipettes with 8-12 M $\Omega$ resistances are used to perform the whole neuron cells under a giga-Ohm seal. Extracellular medium [artificial cerebrospinal fluid $(\mathrm{aCSF})]$ is prepared by mixing $10 \mathrm{mM}$ of 4-(2-hydroxyethyl)-1-piperazineethanesulfonic acid (HEPES), $10 \mathrm{mM}$ of glucose, $2 \mathrm{mM}$ of $\mathrm{CaCl}_{2}$, $140 \mathrm{mM}$ of $\mathrm{NaCl}, 1 \mathrm{mM}$ of $\mathrm{MgCl}_{2}, 3 \mathrm{mM}$ of $\mathrm{KCl}$, and $\mathrm{NaOH}$ into the distilled water at a $p \mathrm{H}$ of 7.4. Internal cellular medium is made by mixing $140 \mathrm{mM}$ of $\mathrm{KCl}, 2 \mathrm{mM}$ of $\mathrm{MgCl}_{2}, 10 \mathrm{mM}$ of HEPE, $10 \mathrm{mM}$ of ethylene glycol-bis( $\beta$ aminoethyl ether)-N,N, $\mathrm{N}^{\prime}, \mathrm{N}^{\prime}$-tetraacetic acid (EGTA), 
$2 \mathrm{mM}$ of $\mathrm{Mg}$-ATP, and $\mathrm{KOH}$ at a $p \mathrm{H}$ of 7.2, and is used to fill the patch pipettes. An Olympus T2 upright microscope and a digital camera are used in the electrophysiology set up to monitor the cells. The stimulations of the human neuroblastoma cell line SH-SY5Y neurons are effective up to 30 min and after that they lose their excitability because of the damage caused by the patched microelectrode.

\section{DISCUSSION}

\section{A. Band alignment in bulk heterojunction solar cell and photoelectrode characterization}

To fabricate a photoelectrode, we use an active layer made of P3HT and PCBM as the donor and acceptor molecules because it is one of the most widely studied and successful photovoltaic blends and has been proven to stimulate neurons grown on the bio-interface (see Supplemental Material [37] Fig. S2) [14,19,21,23]. The bulk heterojunction (BHJ) mechanism has the advantage of separating excitons and reducing the recombination process. Into this blend, we add PbS QDs that can absorb the entire visible and near IR spectral ranges (Appendix A). The use of $\mathrm{PbS}$ QDs in the bulk heterojunction increases the absorbance from 0.15 to 0.17 [Fig. 1(a)], which corresponds to a net absorption increase of $3 \%$ at the wavelength of the blue pump source $(450 \mathrm{~nm})$. The photocurrent enhancement [Fig. 1(b)] is fivefold, significantly above the absorption increase. In order to understand the reason for this, the surface morphology is measured for P3HT:PCBM and P3HT:PbS QDs:PCBM thin films using atomic force microscopy [shown in Figs. 1(c) and 1(d)]. We find that the surface image of the P3HT:PbS QDs:PCBM thin film exhibits smaller intermixed phase-separated domains with better homogeneity and roughness, which is crucial for effective charge separation and collection [37]. In addition, the energy levels of the PbS QDs match with the donor and acceptor molecules for dissociation of the photogenerated excitons (Fig. 2). The energy levels for each photovoltaic material is confirmed by cyclic voltammetry measurements and optical band gap (Appendix A). Hence, the synergistic approach of band alignment, absorption, and enhanced morphology improve the number of charge carriers generated by the photoactive layer to clearly identify the stimulation mechanisms $[42,43]$.

For photoelectrode characterization in physiological media, we fabricate three different photoelectrode configurations with intermediate layers sandwiched between a P3HT:PbS QDs:PCBM bulk heterojunction photoactive layer and a bottom electrode (ITO) [Fig. 2(a)]. We control the charge transfer mechanisms from the substrate to the cells by varying the intermediate layer between the photoactive layer and ITO. For the type I structure in Fig. 2(b), which consists of the photoactive layer on ITO, a dominant Faradaic charge-transfer mechanism is expected because the LUMO energy of the P3HT and PCBM is above the $\mathrm{H}^{+} / \mathrm{H}_{2}$ potential, and (a)

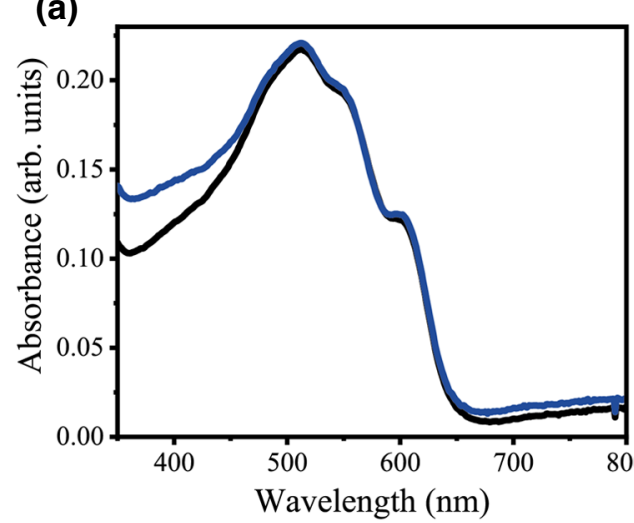

(c)

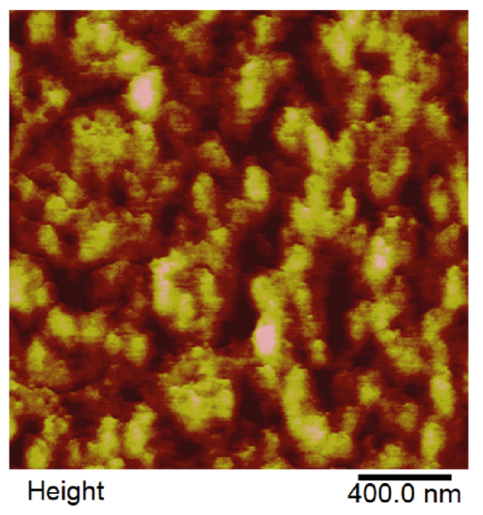

(b)
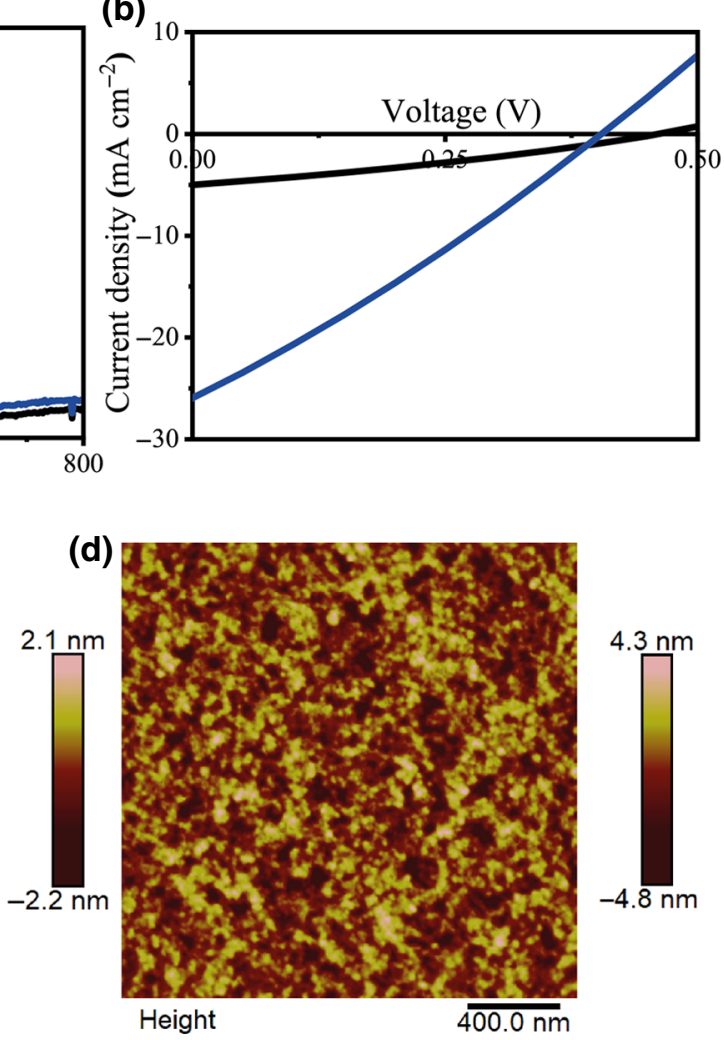

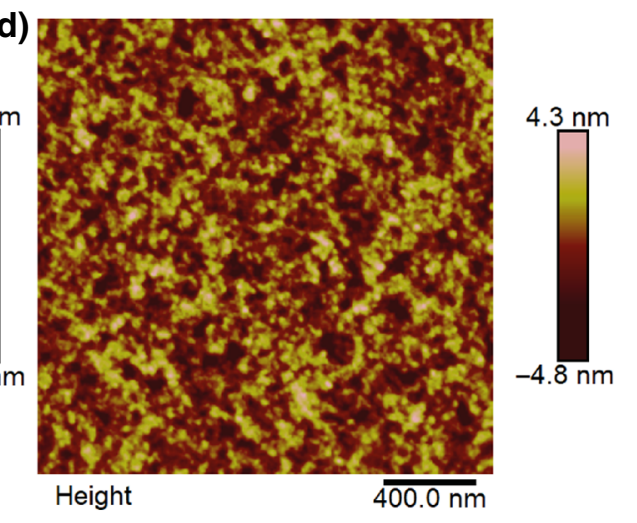

FIG. 1. (a) Ultraviolet-tovisible (UV-Vis) spectrum of P3HT:PCBM (black line) and P3HT:PbS-QDs:PCBM thin films (blue line). (b) Current-voltage characteristics of P3HT:PCBM (black line) and P3HT:PbS-QDs: PCBM (blue line) based solar cells under air-mass $1.5 \mathrm{G}$ solar illumination of $100 \mathrm{~mW} \mathrm{~cm}^{-2}$. Solar cell structures are $\mathrm{ITO} / \mathrm{ZnO} /$ P3HT:PCBM/ $\mathrm{MoO}_{x} / \mathrm{Ag}$ and ITO/ $\mathrm{ZnO} / \mathrm{P} 3 \mathrm{HT}$ :PCBM:PbS-QDs/Mo $\mathrm{O}_{x} / \mathrm{Ag}$. (c) Surface height profile of P3HT:PCBM thin film and (d) P3HT:PbS-QDs:PCBM thin film for $2 \times 2 \mu \mathrm{m}^{2}$ scan area. 


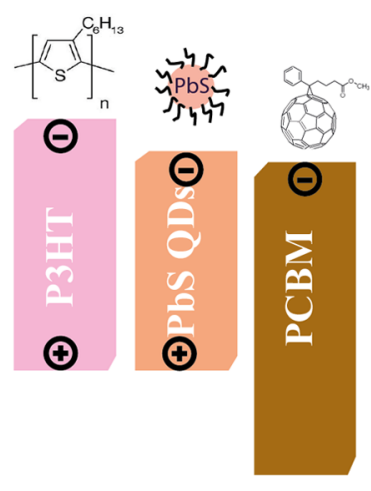

(b)

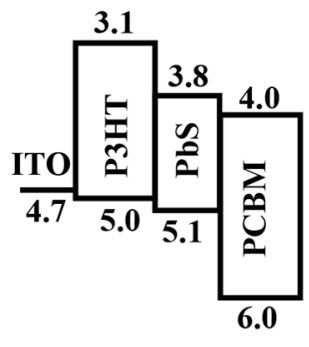

Type I (a)

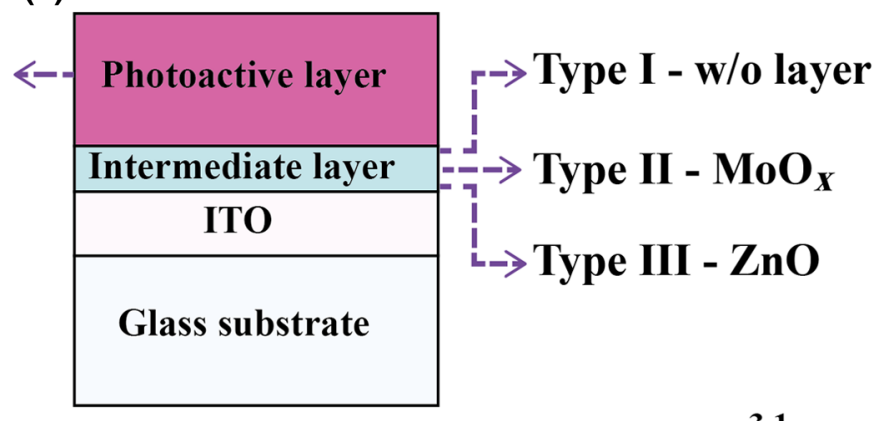

FIG. 2. (a) Photoelectrode device architectures (Types I, II, and III). In all of them, P3HT, $\mathrm{PbS}$ QDs, and PCBM blend is used for the photoactive layer. (b) Energy band diagram for ITO/ P3HT:PbS QDs : PCBM (Type I), (c) ITO $/ \mathrm{MoO}_{x} / \mathrm{P} 3 \mathrm{HT}: \mathrm{PbSQDs}$ : PCBM (Type II), and (d) ITO/ZnO/P3HT:PbS QDs:PCBM (Type III).

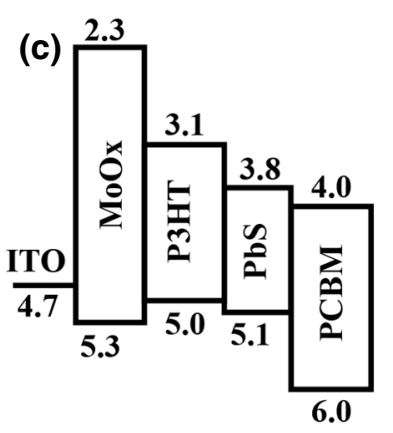

Type II (d)

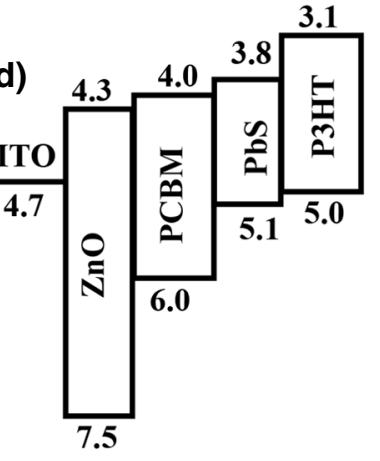

Type III

thus the photogenerated electrons can reduce the water molecules without any bias voltage [44]. To explore the possible Faradaic processes, we investigate the IS under illumination [see Supplemental Material [37] Fig. S2(a)], which can identify electrochemical charge accumulation and transport properties [45-47]. The impedance response of the photoactive layer shows an Ohmic behavior between the high- to mid-frequency ranges [see Supplemental Material [37] Fig. S2(a) inset], and then slowly changes to a depressed semicircle at low frequencies in the complex plane. In the high-frequency region, the bulk characteristic of the device shows a Faradic nature. The impedance response is further simulated using an electrical equivalent circuit to get the physical parameters. We find out that while the charge storage at the electrodeelectrolyte interface has a capacitance of $3.67 \mu \mathrm{F} \mathrm{cm}^{-2}$ in the equivalent circuit (see Supplemental Material [37] Fig. S3), the Faradaic impedance is low $(31 \mathrm{k} \Omega)$ indicating an effective Faradaic charge-transfer mechanism. As expected, the photoelectrode shows a strong Faradaic photocurrent with a peak current level of $12.67 \mathrm{nA}$ under the intensity of $1 \mathrm{~mW} \mathrm{~cm}^{-2}$ and pulse width of $10 \mathrm{~ms}$ [Fig. 3(b)].

One possible way to decrease the Faradaic chargetransfer mechanism can be by partially blocking the electron transport inside the photoelectrode. For that purpose, a $\mathrm{MoO}_{x}$ is introduced in between the photoactive layer and ITO [Fig. 2(c)], which behaves as a potential barrier for electrons. The IS of the photoactive layer with $\mathrm{MoO}_{x}$ also indicates a Faradaic charge-transfer-based current injection, but due to the potential barrier by the $\mathrm{MoO}_{x}$ layer, the Faradaic current is significantly reduced to $0.95 \mathrm{nA}$ [Fig. 3(c)]. This is also supported by the increase of the Faradaic resistance $(82 \mathrm{k} \Omega)$ between the electrode and electrolyte under illumination.

To design an electrode with enhanced capacitive chargetransfer, the carriers need to be localized close to the interface, which will allow strong Coulomb interaction with the ions in the electrolyte. At the same time, these charges need to have an energy level that will not undergo a Faradaic reaction. Since the HOMO energy of the P3HT is above the water oxidation energy, Faradaic process due to hole transfer is limited (see Supplemental Material [37] Fig. S4). Therefore, the holes in the photoactive layer need to be positioned close to the electrode/electrolyte interface. Furthermore, from the above discussed photoelectrodes, we know that the electrons in the photoactive layer can generate a Faradaic reaction. Because of this, the surface electron that can undergo a Faradaic mechanism needs to be moved away from the electrolyte interface and trapped inside the device. For this purpose, we replace the $\mathrm{MoO}_{x}$ layer with a $\mathrm{ZnO}$ layer [Fig. 2(d)], and this type III configuration moves electrons toward the ITO layer and holes toward the electrode/electrolyte interface. Hence, in this structure, holes can effectively attract negative ions in the electrolyte to generate a displacement current.

To analyze the electrochemical behavior of the interface, we perform IS [see Supplemental Material [37] Fig. S2(a)]. The impedance response for the devices under illumination shows a distinctive semicircle in comparison with the Faradaic interfaces between the high- to 

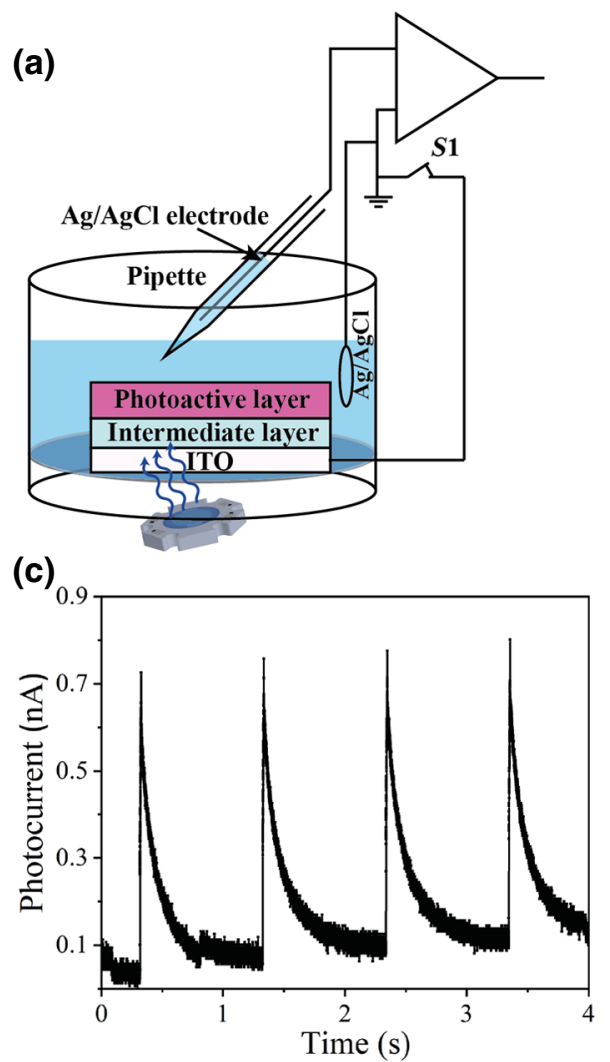
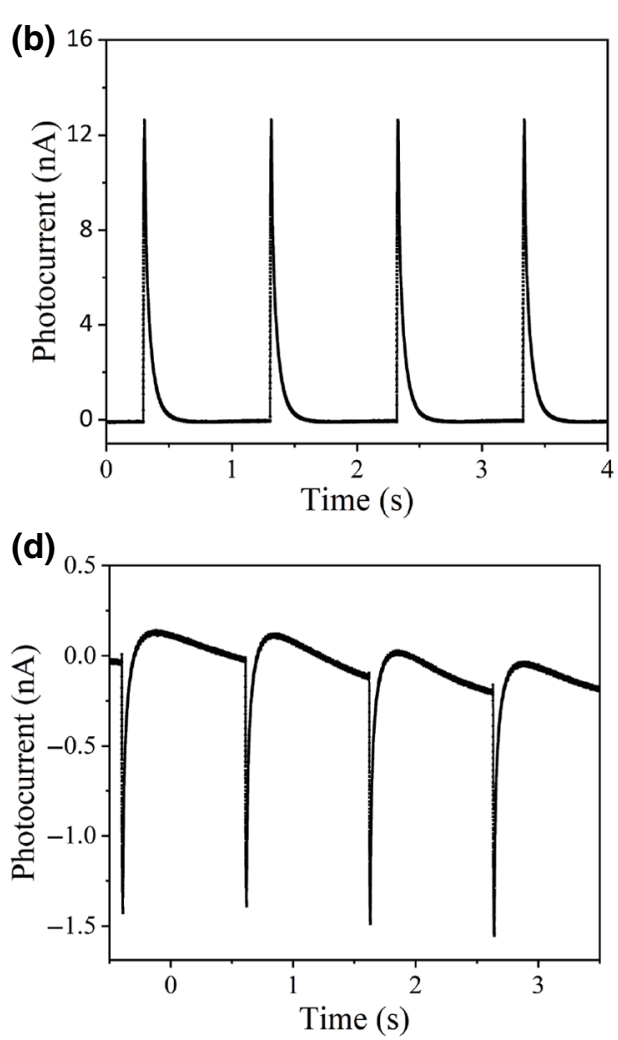

FIG. 3. Photocurrent generated by the photoelectrodes while ITO is grounded. (a) Schematic of the photocurrent measurement set up. The patch pipette is kept close to the surface and the current is measured with a patch-clamp electrode. (b) Photocurrent vs time for ITO/P3HT:PbS-QDs: PCBM photoelectrode (Type I), (c) ITO $/ \mathrm{MoO}_{x} / \mathrm{P} 3 \mathrm{HT}: \mathrm{PbS}-\mathrm{QDs}$ : PCBM photoelectrode (Type II), and (d) ITO $/ \mathrm{ZnO} / \mathrm{P} 3 \mathrm{HT}: \mathrm{PbS}$ QDs:PCBM photoelectrode (Type III). mid-frequency range [See Supplemental Material [37] Fig. S2(a) inset], and then slowly changes to a depressed semicircle at low frequencies in the complex plane. In the high-frequency region, the bulk characteristic of the photoelectrode shows a capacitive nature. The observed capacitance and Faradaic impedance at the interface are found to be $5.47 \mu \mathrm{F} \mathrm{cm}^{-2}$ and $0.16 \mathrm{M} \Omega$, respectively, which are higher than those of the Faradaic devices. These electrochemical properties support the enhanced capacitive nature of the photoelectrode by using $\mathrm{ZnO}$ as the intermediate layer. For further clarification, the Bode modulus and Bode phase are plotted against frequency [see Supplemental Material [37] Fig. S2(b)]. The Bode phase at high frequency is generally interpreted as the electrolyte region, and the region in the $\mathrm{kHz}$ frequency corresponds to the double layer at the photoelectrode/electrolyte interface. At this frequency, we comparatively observe a significantly higher phase angle, which indicates a strong capacitive response.

We measure the photocurrent of the electrode inside the aCSF solution under the same light intensity of $1 \mathrm{~mW} \mathrm{~cm}^{-2}$ and a $10-\mathrm{ms}$ pulse width. Since holes migrate in the direction of P3HT, the direction of the initial spike is reversed in comparison with the Faradaic electrodes. Hence, the hole cloud in the P3HT attracts the negative ions in the electrolyte. Because of the double layer generation on the electrode/electrolyte interface and the additional serial capacitances of the accumulation at the interfacial layer [48], a positive voltage rising between electrolyte and electrode induces a current flow from the electrode toward the electrolyte. After a $10-\mathrm{ms}$ pulse, there is a decreasing slope, which now induces a current generation from the electrolyte to the electrode. The slower kinetics in the displacement current from electrolyte to electrode is possibly due to slower recombination of the carriers in the photoactive layer. Moreover, when we integrate the area under the time-dependent photocurrent, it approximately approaches zero, which also proves that the total number of charges are conserved meaning that the current is generated due to the redistribution of surface charges, not due to either electron or hole tunneling between the electrode and electrolyte. Furthermore, to confirm the key role of band-gap engineering on charge-transfer control, we experimentally test by adding a thin tunneling layer of $1-\mathrm{nm} \mathrm{MoO}_{x}$ between the conductive and photoactive layers or tuning the conductivity by adding a 1-nm gold (Au) layer [see Supplemental Material [37] Fig. S5(a)]. In both cases, Faradaic current is observed [see Supplemental Material [37] Figs. S5(b) and S5(c)], which confirms the importance of band alignment to obtain strong capacitive effects. This approach, which offers an alternative to the variation in physical dimensions, metal doping, and surface chemistry [49], advantageously allows for the design flexibility such that after the optimization of the photoactive layer for the effective transduction of photons to charge carriers, the inner layers 

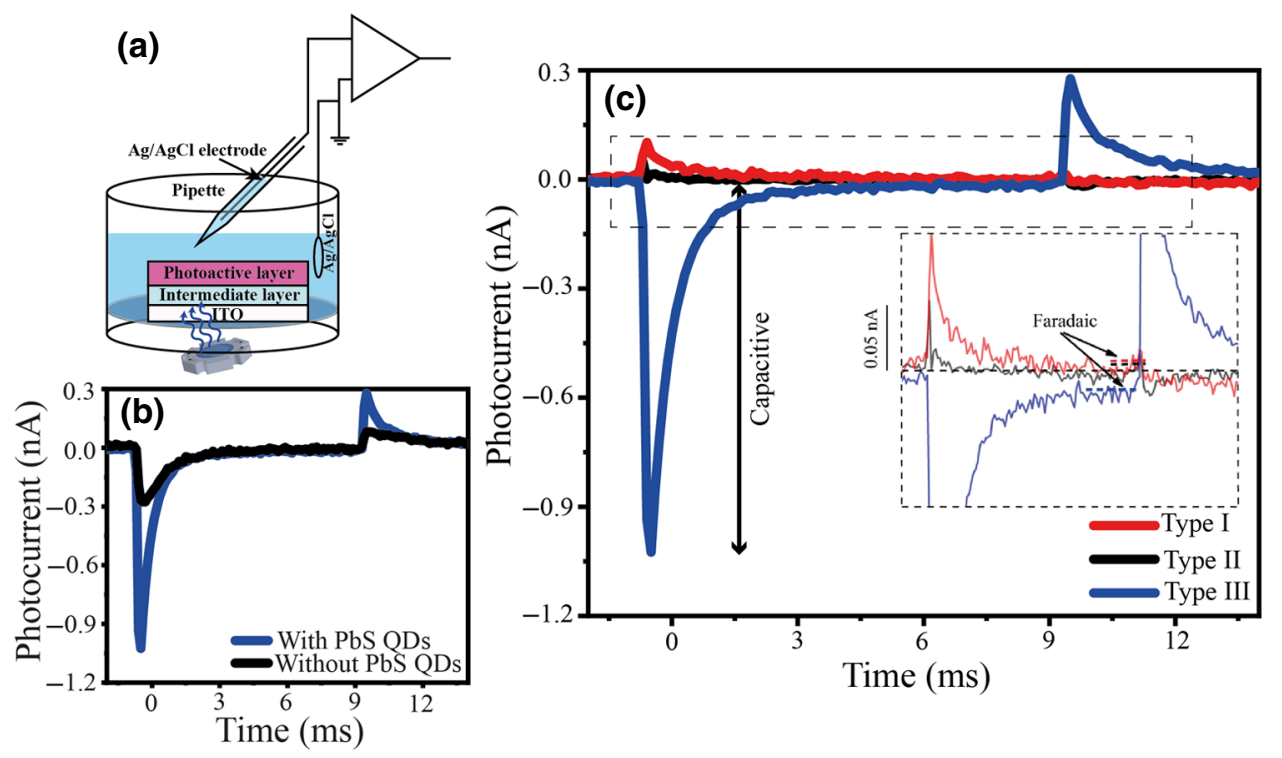

FIG. 4. (a) Photocurrent measurement configuration in freestanding mode. aCSF solution is grounded with $\mathrm{Ag} / \mathrm{AgCl}$ reference electrode. (b) Photocapacitive current levels of ITO/ $\mathrm{ZnO} / \mathrm{P} 3 \mathrm{HT}$ :PCBM and ITO/ ZnO/P3HT:PbS-QDs:PCBM pho toelectrodes. (c) Capacitive and Faradaic components of type I, type II, and type III photoelectrodes under illumination of 10-ms light pulses with an intensity of $1 \mathrm{~mW} \mathrm{~cm}^{-2}$. can be engineered for optimized and targeted stimulation mechanisms.

\section{B. Charge-transfer mechanism in biological media in a wireless, free-standing mode}

We next investigate charge-transfer mechanisms in biological media in a free-standing mode close to the realistic situation of a neural stimulation condition. In this case, the photocurrent is measured with a micropipette electrode close to the photoelectrode in aCSF solution. In this setup configuration, the bath solution is grounded with an $\mathrm{Ag} / \mathrm{AgCl}$ reference electrode without any wiring to the ITO electrode [Fig. 4(a)]. This set up reflects the working conditions of a standalone implantable device. We measure the photoresponses of type I, type II, and type III photoelectrodes in this mode under blue LED light pulses having an intensity of $1 \mathrm{~mW} \mathrm{~cm}{ }^{-2}$ and a pulse width of $10 \mathrm{~ms}$ [Fig. 4(b)] and quantitatively analyze the contribution by the capacitive and Faradaic components using the approach of Jiang et al. [49]. The type III photoelectrode is specifically designed for strong capacitive photocurrents and it exhibits a capacitive current level of $1.02 \mathrm{nA}$, which is at least 10 -fold higher than the other photoelectrodes. The Faradaic photocurrent is less than $0.5 \%$ for the type III photoelectrode, which is almost negligible, showing that the major contribution comes from the capacitive photocurrent [Fig. 4(c) inset]. In this mode, the Faradaic currents by type I and type II are significantly lower and a low level of capacitive photocurrents that correspond to 0.10 and $0.05 \mathrm{nA}$ are observed, respectively. Due to the effective collection of holes in the photoactive layer in the type III photoelectrode, the capacitive current is also controllably reversed in comparison with the other photoelectrodes. Just as in the solar cell measurements, the addition of PbS QDs to type III photoelectrodes enhances the photocurrent by approximately four times.

\section{Neuronal photostimulation}

Initially, we check the biocompatibility of the photoelectrodes and we observe that they have comparable cell viability with the ITO control. These results suggest that the photoelectrodes do not exhibit any toxic effect on the cells (Appendix B). Patch clamping in whole-cell configuration in the current clamp $(I=0)$ is used to record cellular electrophysiology [Fig. 5(a)] both for dark and under light conditions. Figure 5(b) shows a typical $I V$ characteristic of a SH-SY5Y cell, which is measured with a voltage clamp in dark condition. The cell has a quasilinear response around the resting potential, as assumed in the two-domain-stimulation model (Appendix C). Just as in photocurrent experiments, light pulses with an intensity level of $1 \mathrm{~mW} \mathrm{~cm}^{-2}$ and a $10-\mathrm{ms}$ pulse width are used for the photostimulation experiments. The electrophysiology experiment is performed in wireless, free-standing mode as explained in Sec. B.

Furthermore, organic substrates may also stimulate the neurons via photothermal or thermocapacitive effects, which induces a depolarization due to phase transition in the phospholipid ordering and afterward ignites a hyperpolarization due to the variation of the membrane conductance in the channels and rearrangement of the ions [50,51]. To investigate the contribution of the thermal effect, we calculate the temperature produced by light illumination. The maximum temperature that can be induced for $1 \mathrm{~mW} \mathrm{~cm}{ }^{-2}$ is less than $0.01^{\circ} \mathrm{C}$ (Appendix D), which is insufficient for photothermal stimulation of the cells. Moreover, the level of intensities that we use in this study is comparatively two-orders of magnitude lower than the reports studying the thermal activation of neurons [26]. 
(a)

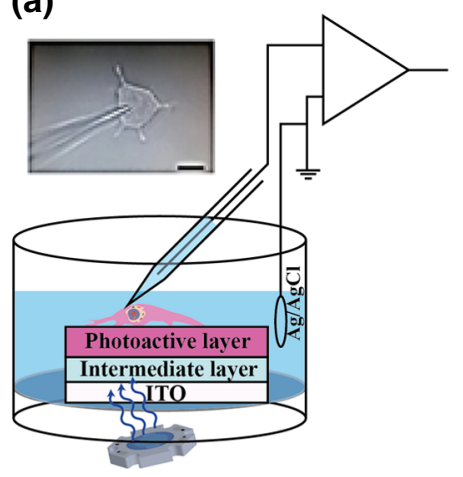

(b)

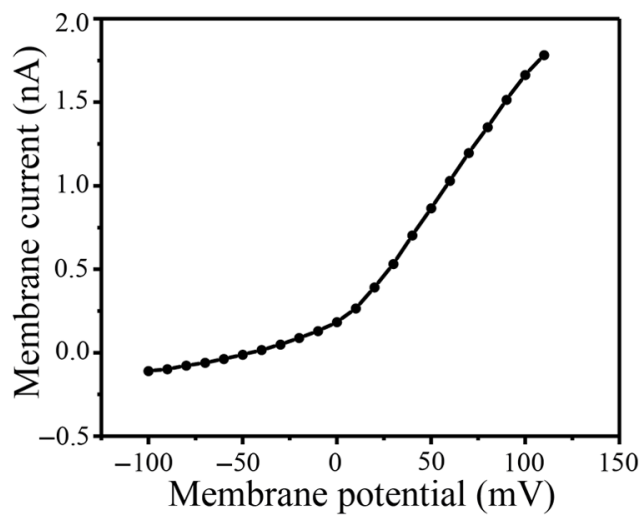

(c)

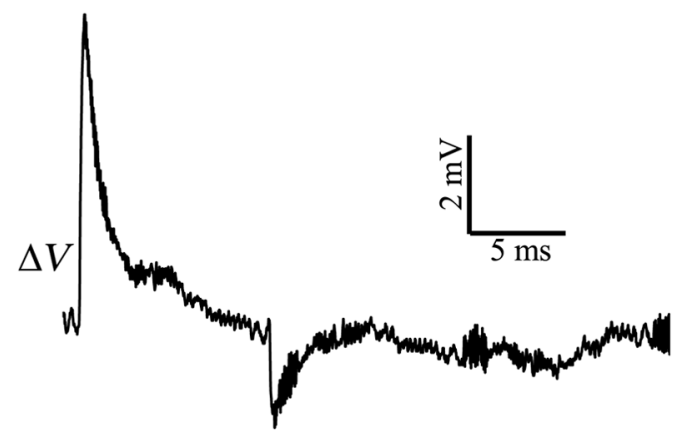

(d)

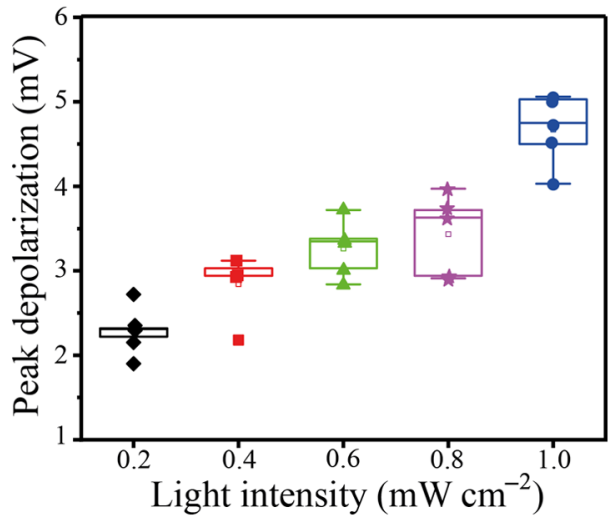

FIG. 5. Analysis of SH-SY5Y cell responses to 10 -ms light pulses on type-III photoelectrode architecture (ITO/ZnO/P3HT: PbS-QDs: PCBM). (a) Schematic of the whole-cell patchclamp recoding configuration of the photoelectrode in freestanding mode. The cells are grown on the photoelectrode and adhere on the polymer surface. A LED at far field is used to illuminate and activate the photoelectrode. (b) Currentvoltage characteristics of a typical SH-SY5Y cell under dark. (c) Membrane potential variation upon light illumination $(10 \mathrm{~ms}$, $1 \mathrm{~mW} \mathrm{~cm}{ }^{-2}$ ). (d) Peak depolarization recorded for different light intensities $(N=5)$.
Even though the photoelectrochemical currents may not be appropriate for long-term neural stimulation, they can be useful for short-term stimulation [52].

Alternatively, the capacitive charge-transfer mechanism presents a safe stimulation strategy by redistribution of the ion concentration in the electrolyte, which can be strongly generated by the type III photoelectrode. Figure 5(c) shows the membrane potential response by the type III photoelectrode after illumination of light pulses showing an initial depolarization (positive spike) of the cell membrane followed by a slower transient repolarization. When the light is switch off after $10 \mathrm{~ms}$, a complementary behavior of a negative spike is recorded followed by a transient hyperpolarization before the cell membrane reaches the resting potential. This depolarization and hyperpolarization are an expected behavior due to the bidirectional current generation for each pulse during the rising and falling times of the light intensity. The peak depolarization for different illumination intensities are measured and at $0.2 \mathrm{~mW} \mathrm{~cm}{ }^{2}$ intensity levels can still lead to significant peak membrane depolarization [Fig. 5(d)]. It is also evident from the box plot that peak depolarization has variations from cell to cell and can be increased by increasing the light intensity under safe neural stimulation limits.

\section{CONCLUSION}

In summary, without changing the surface material of the photoelectrode, we demonstrate that the band engineering of the photoelectrodes enables control of the strength and type of photostimulation mechanisms. Via the proper selection of the intermediate layer, the strength of the Faradaic charge transfer can be adjusted and can even be drastically suppressed and converted to a capacitive mechanism. This approach is different from the conventional one that modifies the cell-electrode interaction by changing the material on the surface [9]. Moreover, the incorporation of QDs into the organic bulk-heterojunction blend significantly enhances the number of photogenerated charge carriers that enables to identify the Faradaic and capacitive charge-transfer mechanisms. In perspective, utilization of controlling the photostimulation mechanism provides an alternative opportunity for neural prosthesis. Some important challenges in neural prosthesis, such as Faradaic degradation because of irreversible reactions at the photoelectrode-neuron interface, long term biocompatibility, and finding a compatible material can be solved. Therefore, the results point to an alternative direction in engineering of neural interfaces for effective and safe photostimulation.

\section{ACKNOWLEDGMENTS}

This project has received funding from the European Research Council (ERC) under the European Union's Horizon 2020 Research and Innovation Programme (Grant No. 639846). 
(a)

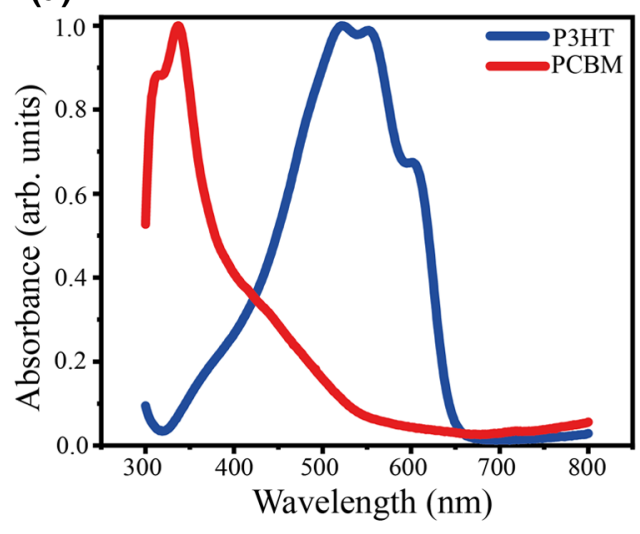

(b)

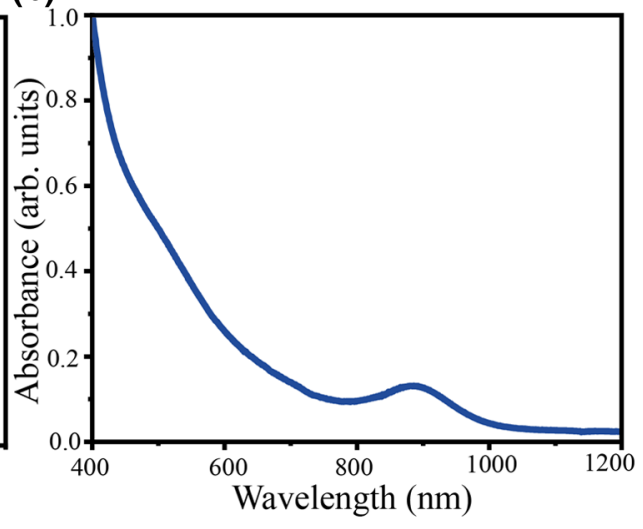

FIG. 6. (a) Absorbance of photovoltaic materials $\mathrm{P} 3 \mathrm{HT}$ and PCBM. (b) Absorption spectra of $\mathrm{PbS}$ colloidal QDs in toluene solvent. (c) Cyclic voltammogram of photovoltaic materials (P3HT, PCBM, PbS QDs) with respect to Ferrocene $\left(\mathrm{Fc} / \mathrm{Fc}^{+}\right)$as the internal reference.

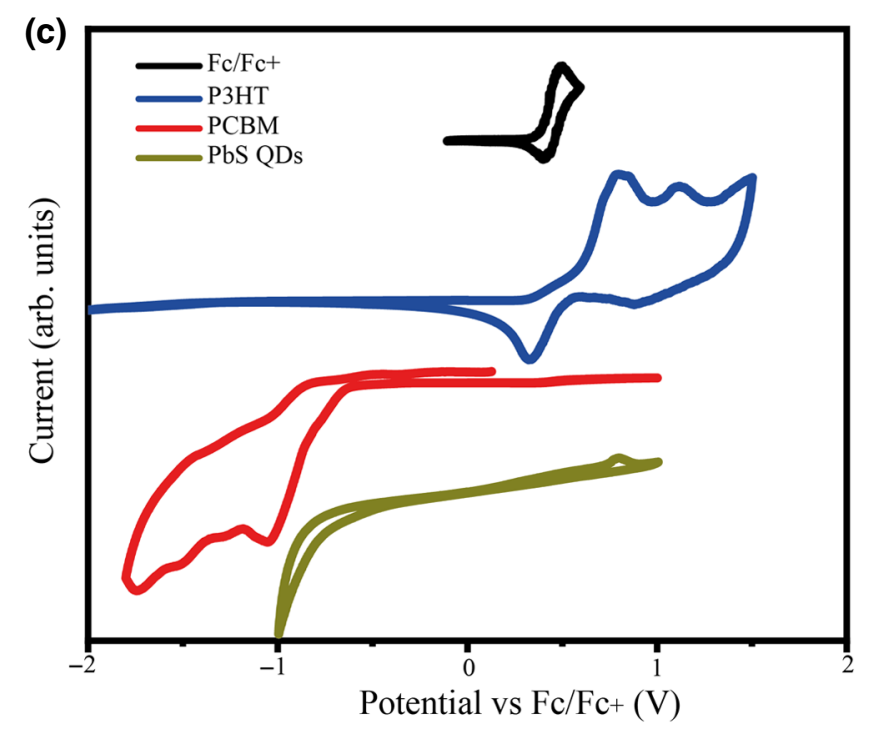

\section{APPENDIX A: ENERGY LEVEL CALCULATION OF PHOTOVOLTAIC MATERIALS}

The energy levels of the photovoltaic materials (P3HT, PCBM, and PbS QDs) are determined by cyclic voltammetry (CV) experiments. In this experiment, a $0.1-\mathrm{M}$ tetrabutylammonium hexafluorophosphate (TBAPF6) supporting electrolyte is dissolved in anhydrous acetonitrile solvent to make an electrolyte solution. Next, we add ferrocene in the electrolyte as an internal reference. For $\mathrm{CV}$ measurement, a three-electrode set up consisting of $\mathrm{Ag} / \mathrm{AgCl}$ as the reference electrode, platinum wire as the counter electrode, and thin films of photovoltaic materials as the working electrode are used. The HOMO and LUMO levels can be estimated by oxidation $\left(E_{\mathrm{ox}}^{\text {onset }}\right)$ and reduction onset $\left(E_{\text {red }}^{\text {onset }}\right)$ potentials of the materials using the following equations

$$
\begin{aligned}
& E_{\mathrm{HOMO}}=-e\left(4.8-E_{\mathrm{Fc} / \mathrm{Fc}^{+}}+E_{\mathrm{ox}}^{\mathrm{onset}}\right), \\
& E_{\mathrm{LUMO}}=-e\left(4.8-E_{\mathrm{Fc} / \mathrm{Fc}^{+}}+E_{\text {red }}^{\text {onset }}\right),
\end{aligned}
$$

where, $E_{\mathrm{Fc} / \mathrm{Fc}^{+}}$is the half-wave potential and is calculated as $0.11 \mathrm{~V}$ from the redox potential of ferrocene. During CV measurement, the redox reactions may not be observed together for each material. Hence, we calculate an optical band gap of $1.92 \mathrm{eV}$ for P3HT, $2.03 \mathrm{eV}$ for PCBM, and $1.25 \mathrm{eV}$ for PbS QDs using an absorbance plot [Figs. 6(a) and 6(b)]. The levels are calculated using Eqs $\mathrm{A} 1$ and $\mathrm{A} 2$ and the optical band gap $\left(E_{g}\right)$ and HOMO/LUMO are determined as $-5.01 /-3.09 \mathrm{eV}$ for P3HT, $-6.04 /-4.01 \mathrm{eV}$ for PCBM, and $-5.11 /-3.85 \mathrm{eV}$ for PbS QDs.

\section{APPENDIX B: CELL VIABILITY ASSAY \\ 1. Cell growth}

The SH-SY5Y cell line is used throughout the experiments. SH-SY5Y cells are cultured in Dulbecco's Modified Eagle's Medium (DMEM, Gibco 21969-035) supplemented with $10 \%$ fetal bovine serum (FBS, Gibco 10500), 1\% L-glutamine (Gibco, 25030-081), and 1\% penicillin-streptomycin (Gibco 15240-062). Cultures are maintained at $37^{\circ} \mathrm{C}$ in a $5 \% \mathrm{CO}_{2}, 85 \%$ humidified 


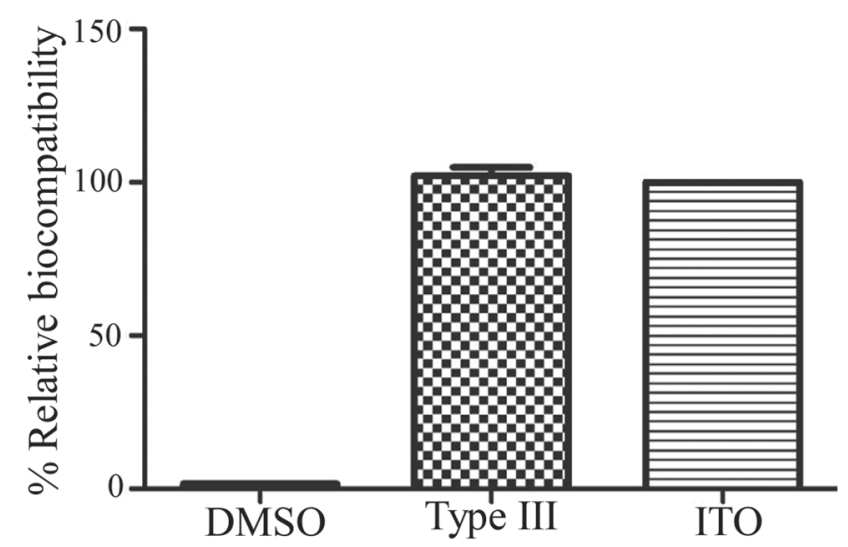

FIG. 7. The cell viability assessment of photoelectrodes on the SH-SY5Y cell line. The effect of the photoelectrodes on metabolic activity of SH-SY5Y cells assessed by MTT. Data is presented in a column graph plotting the mean with the standard error of the mean (SEM). An unpaired two-tailed $t$ test is performed to determine level of significance. ${ }^{*} p<0.05$ is considered as statistically significant and nonsignificant differences are presented as "ns."

incubator. Cells are passaged and supplied with fresh medium every 2-3 days.

\section{MTT assay}

Cytotoxicity assay is performed as described by Bahmani Jalali et al. [53]. Briefly, photoelectrodes are cut into $0.40 \times 0.40 \mathrm{~cm}^{2}$ pieces then sterilized by $70 \%$ ethanol treatment and 30-min ultraviolet (UV) light exposure. Sterilized substrates are placed into a sterile 96-well plate and $1 \times 10^{4}$ SH-SY5Y cells are seeded onto substrates in $200 \mu \mathrm{l}$ of growth medium. The plate is incubated for $48 \mathrm{~h}$ in an incubator under $37^{\circ} \mathrm{C}$ in the presence of $5 \% \mathrm{CO}_{2}$. Then, the growth medium is replaced with DMEM containing $1 \mathrm{mg} / \mathrm{ml}$ 3-(4,5-Dimethylthiazol-2-yl)2,5-diphenyltetrazolium bromide (MTT) solution $(100 \mu \mathrm{l})$ and further incubated for $4 \mathrm{~h}$ at $37^{\circ} \mathrm{C}$ to allow formazan formation. Subsequently, the MTT solution is removed and formazan salt is dissolved in ethyl alcohol/dimethyl sulfoxide (DMSO) $(200 \mu \mathrm{l})$. The amounts of formazan are measured with a microplate reader (BioTek, Synergy H1) in a clean 96-well plate. The relative cell viability is calculated as

$$
\text { viability }=\left(\mathrm{OD}_{\text {sample }} / \mathrm{OD}_{\text {control }}\right) \times 100 \text {. }
$$

The optical density of sample, $\mathrm{OD}_{\text {sample }}$ is obtained from the photoelectrodes and the optical density of control sample, $\mathrm{OD}_{\text {control }}$ is obtained from the ITO control. The experiment is performed with at least three biological replicates and each biological replicate consists of nine technical replicates $(n=3)$.

\section{Biocompatibility statistics}

After the synthesis of the photoelectrodes, we next investigate its effect on cell viability and cell growth of SH-SY5Y cells using MTT assay and tracking assay. As
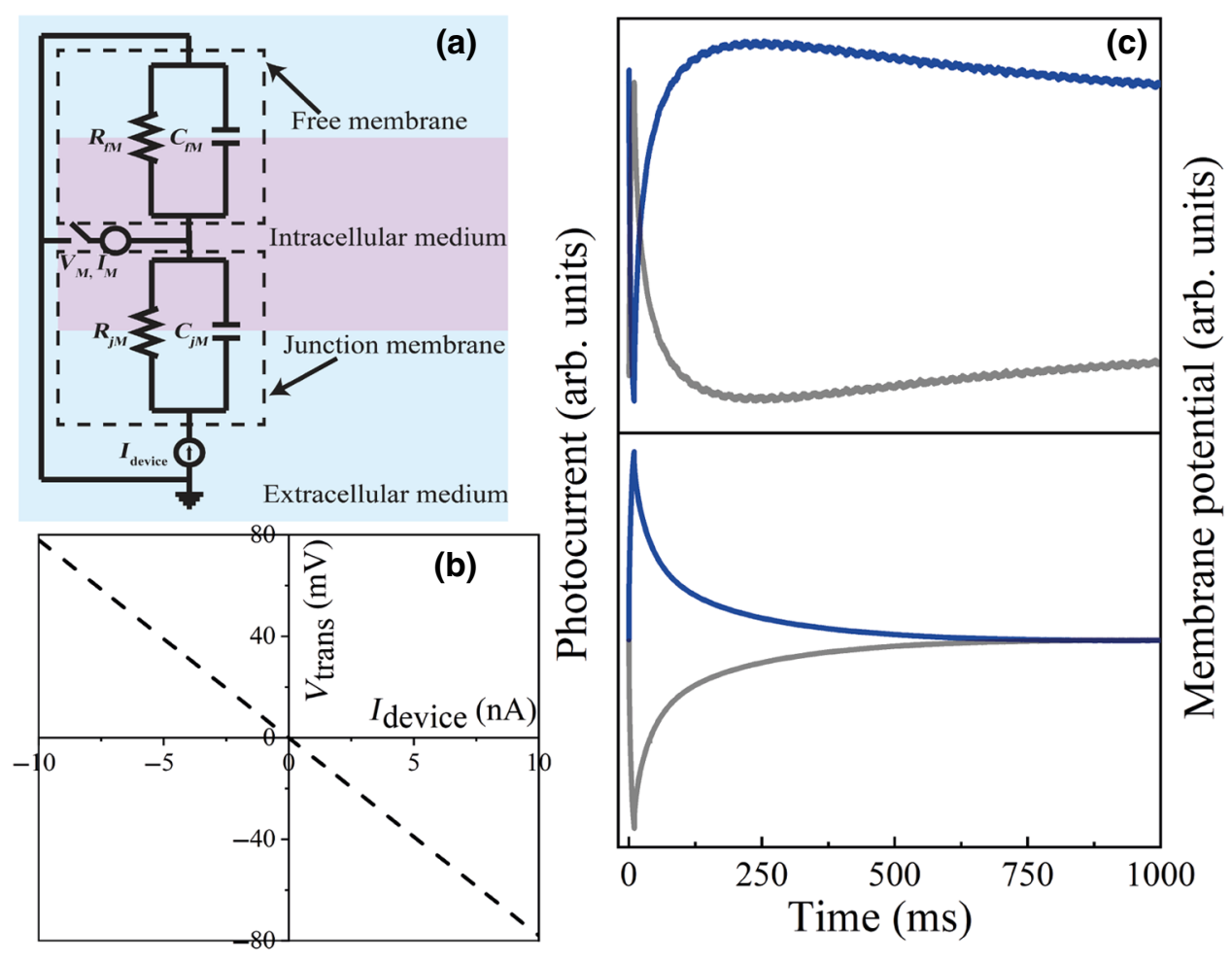

FIG. 8. (a) Two-domain circuit representation of neuronal cell membrane in subthreshold condition. Blue part shows the extracellular region and the reddish part shows the intracellular region. (b) Simulated transmembrane potential under extracellular input current $\left(I_{\text {device }}\right)$. The potential shows a linear response as a result of the injected current. (c) The output transmembrane potential waveforms (gray lines) as a response to the capacitive (top) and Faradaic (bottom) current waveforms that are used as the input (blue lines). 
can be seen in Fig. 7, cells grown on substrates have comparable viability compared with the ITO control rather than the $10 \%$ DMSO treated cell samples. These results suggest the substrate did not produce any toxic effect on the cells.

\section{APPENDIX C: TWO-DOMAIN-STIMULATION MODEL}

Neuron membrane potential is controlled by the inflow and outflow of ions, and to understand the effect of extracellular stimulation by the photoactive substrates on a nerve cell, we use a two-domain-stimulation model [10], which considers the polarization of the attached membrane on the substrate and free membrane. The circuit diagram of the two-domain-stimulation model is shown in Fig. 8(a). Here, we assume the equivalent circuit of the cell membrane as an $R C$ circuit instead of a Hodgkin-Huxley model. This is due to the use of undifferentiated SH-SY5Y cells in our electrophysiology experiments, which generally operate under the subthreshold regime. Moreover, we model the photoactive substrates as current sources that can generate current flow via Faradaic and capacitive mechanisms. Since we measure the current levels of each substrate on its surface with a distance between the patch pipette and the surface, in the equivalent circuit, the effect of the leakage current due to a cleft between a cell and substrate is assumed to be included in the current source. By writing the circuit via Kirchhoff's current law at an intracellular medium node, the equation can be expressed as

$$
A_{f m} C_{m} \frac{d V_{m}}{d t}+A_{f m} \frac{V_{m}}{R_{m}}+A_{j m} I_{\text {device }}=0
$$

where $V_{m}$ is the free membrane potential with respect to ground, $C_{m}=C_{f m} / A_{f m}=C_{j m} / A_{j m}$ is cell membrane capacitance, where $C_{j m}$ is the junction membrane capacitance, $C_{f m}$ is the free membrane capacitance, $R_{m}=$ $R_{f m} \times A_{f m}=R_{j m} \times A_{j m}$ is the membrane resistance, $A_{j m}$ is attached membrane area on the electrode, $A_{f m}$ is the free membrane area, $R_{j m}$ is the junction membrane resistance, $R_{f m}$ is the free membrane resistance, and $I_{\text {device }}$ is the stimuli current generated by the photoactive surfaces. We also know that while a cell is not stimulated by an extracellular electrode, it will remain at its resting potential $\left(V_{\text {rest }}\right)$, and thus, the initial and steady-state boundary conditions of the membrane potential are $V_{\text {rest }}$.

The general solution of Eq. (C1) is given by

$$
V_{m}=V_{\text {rest }}-\frac{A_{j m}}{A_{f m}} R_{m} I_{\text {device }}\left(1-e^{\left[-t /\left(R_{m} C_{m}\right)\right]}\right) .
$$

The transmembrane potential with respect to the resting potential can be written as

$$
V_{\text {trans }}=V_{m}-V_{\text {rest }}=-\frac{A_{j m}}{A_{f m}} R_{m} I_{\text {device }}\left(1-e^{\left[-t /\left(R_{m} C_{m}\right)\right]}\right) .
$$

We simulate the current-transmembrane potential for a single cell via extracellular excitation. Due to the linearity of a $R C$ circuit, we observe a linear response for different current injection levels [Fig. 8(b)]. The physical interpretation of this result is that higher current will lead to stronger depolarization and hyperpolarization. Moreover, we also apply Faradaic and capacitive current waveforms into the model. For Faradaic currents, we expect a hyperpolarizing behavior and relaxation to the resting state. For the capacitive currents, a depolarization is expected to be followed by a hyperpolarization [Fig. 8(c)].

\section{APPENDIX D: THERMAL EFFECT AT NEURON SURFACE DUE TO LIGHT ILLUMINATION}

From the thermodynamic relation,

$$
\begin{aligned}
d_{q}(\mathrm{rev}, p) & =m C_{p} d T \\
\int_{0}^{q} d q(\mathrm{rev}, p) & =m C_{p} \int_{295 \mathrm{~K}}^{T} d T .
\end{aligned}
$$

Considering the water heat capacity $C_{p}=75.291 \mathrm{~J} \mathrm{~K}^{-1}$ $\mathrm{mol}^{-1}$, we can calculate specific heat capacity as

$$
q=m C_{p} \Delta T
$$

On the other hand, we can find the thermal energy $(q)$ because of light illumination

$$
q=I \times t=1 \mathrm{~s} \times 1 \mathrm{~mW}=1 \times 10^{-3} \mathrm{~J},
$$

where $I$ is the light illumination power equal to $1 \mathrm{~mW}$ and $t$ is illumination time, $1 \mathrm{~s}$.

From Eqs. (D3) and (D4)

$$
\begin{aligned}
1 \times 10^{-3} \mathrm{~J}= & (0.0332 \mathrm{~g} / 18 \mathrm{~g} / \mathrm{mol}) \\
& \times 75.291 \mathrm{~J} \mathrm{~K}^{-1} \mathrm{~mol}^{-1} \Delta T \\
\Delta T= & 0.00728^{\circ} \mathrm{C} .
\end{aligned}
$$

[1] L. Bareket-Keren and Y. Hanein, Novel interfaces for light directed neuronal stimulation: advances and challenges, Int. J. Nanomedicine 9(Suppl 1), 65 (2014).

[2] F. Di Maria, F. Lodola, E. Zucchetti, F. Benfenati, and G. Lanzani, The evolution of artificial light actuators in living 
systems: from planar to nanostructured interfaces, Chem. Soc. Rev. 47, 4757 (2018).

[3] A. Steude, E. C. Witts, G. B. Miles, and M. C. Gather, Arrays of microscopic organic LEDs for high-resolution optogenetics, Sci. Adv. 2, e1600061 (2016).

[4] V. Nikolenko, K. E. Poskanzer, and R. Yuste, Two-photon photostimulation and imaging of neural circuits, Nat. Methods 4, 943 (2007).

[5] J. Rivnay, H. Wang, L. Fenno, K. Deisseroth, and G. G. Malliaras, Next-generation probes, particles, and proteins for neural interfacing, Sci. Adv. 3, e1601649 (2017).

[6] S. Szobota and E. Y. Isacoff, Optical control of neuronal activity, Annu. Rev. Biophys. 39, 329 (2010).

[7] E. Zrenner, K. U. Bartz-Schmidt, H. Benav, D. Besch, A. Bruckmann, V.-P. Gabel, F. Gekeler, U. Greppmaier, A. Harscher, S. Kibbel, J. Koch, A. Kusnyerik, T. Peters, K. Stingl, H. Sachs, A. Stett, P. Szurman, B. Wilhelm, and R. Wilke, Subretinal electronic chips allow blind patients to read letters and combine them to words, Proc. R. Soc. B: Biol. Sci. 278, 1489 (2010).

[8] X. Han and E. S. Boyden, Multiple-color optical activation, silencing, and desynchronization of neural activity, with single-spike temporal resolution, PLoS One 2, e299 (2007).

[9] S. F. Cogan, Neural stimulation and recording electrodes, Annu. Rev. Biomed. Eng. 10, 275 (2008).

[10] I. Schoen and P. Fromherz, The mechanism of extracellular stimulation of nerve cells on an electrolyte-oxidesemiconductor capacitor, Biophys. J. 92, 1096 (2007).

[11] G. G. Wallace, S. E. Moulton, and G. M. Clark, ElectrodeCellular Interface, Science 324, 185 (2009).

[12] E. Pastrana, Optogenetics: controlling cell function with light, Nat. Methods 8, 24 (2010).

[13] D. Boinagrov, X. Lei, G. Goetz, T. I. Kamins, K. Mathieson, L. Galambos, J. S. Harris, Jr., and D. Palanker, Photovoltaic Pixels for Neural Stimulation: Circuit Models and Performance, IEEE Trans. Biomed. Circuits Syst. 10, 85 (2016).

[14] E. Mosconi, P. Salvatori, M. I. Saba, A. Mattoni, S. Bellani, F. Bruni, B. Santiago Gonzalez, M. R. Antognazza, S. Brovelli, G. Lanzani, H. Li, J.-L. Brédas, and F. De Angelis, Surface polarization drives photoinduced charge separation at the P3HT/water interface, ACS Energy Lett. 1, 454 (2016).

[15] F. Benfenati and G. Lanzani, New technologies for developing second generation retinal prostheses, Lab Anim. (NY) 47, 71 (2018).

[16] D. R. Merrill, M. Bikson, and J. G. R. Jefferys, Electrical stimulation of excitable tissue: design of efficacious and safe protocols, J. Neurosci. Methods 141, 171 (2005).

[17] L. Bareket-Keren and Y. Hanein, Carbon nanotube-based multi electrode arrays for neuronal interfacing: Progress and prospects, Front. Neural Circuits 6, 122 (2012).

[18] G. Simone, D. Di Carlo Rasi, X. de Vries, G. H. L. Heintges, S. C. J. Meskers, R. A. J. Janssen, and G. H. Gelinck, Near-infrared tandem organic photodiodes for future application in artificial retinal implants, Adv. Mater. 30, 1804678 (2018).

[19] D. Ghezzi, M. R. Antognazza, R. Maccarone, S. Bellani, E. Lanzarini, N. Martino, M. Mete, G. Pertile, S. Bisti, G. Lanzani, and F. Benfenati, A polymer optoelectronic interface restores light sensitivity in blind rat retinas, Nat. Photonics 7, 400 (2013).

[20] V. Gautam, D. Rand, Y. Hanein, and K. S. Narayan, A polymer optoelectronic interface provides visual cues to a blind retina, Adv. Mater. 26, 1751 (2013).

[21] D. Ghezzi, M. R. Antognazza, M. Dal Maschio, E. Lanzarini, F. Benfenati, and G. Lanzani, A hybrid bioorganic interface for neuronal photoactivation, Nat. Commun. 2, 166 (2011).

[22] V. Gautam, M. Bag, and K. S. Narayan, Single-pixel, single-layer polymer device as a tricolor sensor with signals mimicking natural photoreceptors, J. Am. Chem. Soc. 133, 17942 (2011).

[23] M. R. Antognazza, D. Ghezzi, D. Musitelli, M. Garbugli, and G. Lanzani, A hybrid solid-liquid polymer photodiode for the bioenvironment, Appl. Phys. Lett. 94, 243501 (2009).

[24] C. Sekirnjak, P. Hottowy, A. Sher, W. Dabrowski, A. Litke, and E. Chichilnisky, Electrical stimulation of mammalian retinal ganglion cells with multielectrode arrays, J. Neurophysiol. 95, 3311 (2006).

[25] M. G. Shapiro, K. Homma, S. Villarreal, C.-P. Richter, and F. Bezanilla, Infrared light excites cells by changing their electrical capacitance, Nat. Commun. 3, 736 (2012).

[26] N. Martino, P. Feyen, M. Porro, C. Bossio, E. Zucchetti, D. Ghezzi, F. Benfenati, G. Lanzani, and M. R. Antognazza, Photothermal cellular stimulation in functional bio-polymer interfaces, Sci. Rep. 5, 8911 (2015).

[27] T. Rose and L. Robblee, Electrical stimulation with Pt electrodes. VIII. Electrochemically safe charge injection limits with $0.2 \mathrm{~ms}$ pulses (neuronal application), IEEE Trans. Biomed. Eng. 37, 1118 (1990).

[28] M. Schaldach, M. Hubmann, A. Weikl, and R. Hardt, Sputter-deposited TiN electrode coatings for superior sensing and pacing performance, Pacing Clin. Electrophysiol. 13, 1891 (1990).

[29] M. Tykocinski, Y. Duan, B. Tabor, and R. S. Cowan, Chronic electrical stimulation of the auditory nerve using high surface area (HiQ) platinum electrodes, Hear. Res. 159, 53 (2001).

[30] S. Venkatraman, J. Hendricks, Z. A. King, A. J. Sereno, S. Richardson-Burns, D. Martin, and J. M. Carmena, In vitro and in vivo evaluation of PEDOT microelectrodes for neural stimulation and recording, IEEE Trans. Neural Syst. Rehabil. Eng. 19, 307 (2011).

[31] P. Fromherz and A. Stett, Silicon-Neuron Junction: Capacitive Stimulation of an Individual Neuron on a Silicon Chip, Phys. Rev. Lett. 75, 1670 (1995).

[32] A. G. Pattantyus-Abraham, I. J. Kramer, A. R. Barkhouse, X. Wang, G. Konstantatos, R. Debnath, L. Levina, I. Raabe, M. K. Nazeeruddin, M. Grätzel, and E. H. Sargent, Depleted-heterojunction colloidal quantum dot solar cells, ACS Nano 4, 3374 (2010).

[33] S. A. McDonald, G. Konstantatos, S. Zhang, P. W. Cyr, E. J. D. Klem, L. Levina, E. H. Sargent, I. J. Kramer, A. R. Barkhouse, X. Wang, G. Konstantatos, R. Debnath, L. Levina, I. Raabe, M. K. Nazeeruddin, M. Grätzel, and E. H. Sargent, Depleted-heterojunction colloidal quantum dot solar cells, Nat. Mater. 4, 138 (2005).

[34] R. J. Ellingson, M. C. Beard, J. C. Johnson, P. Yu, O. I. Micic, A. J. Nozik, A. Shabaev, and A. L. Efros, Highly 
efficient multiple exciton generation in colloidal $\mathrm{PbSe}$ and PbS quantum dots, Nano Lett. 5, 865 (2005).

[35] F. Xi, F. Guojia, Q. Pingli, S. Nanhai, L. Nishuang, Z. Qiao, C. Fei, Y. Longyan, and Z. Xingzhong, Deposition temperature effect of RF magnetron sputtered molybdenum oxide films on the power conversion efficiency of bulk-heterojunction solar cells, J. Phys. D: Appl. Phys. 44, 045101 (2011).

[36] W. J. D. Beenken, F. Herrmann, M. Presselt, H. Hoppe, S. Shokhovets, G. Gobsch, and E. Runge, Sub-bandgap absorption in organic solar cells: experiment and theory, Phys. Chem. Chem. Phys. 15, 16494 (2013).

[37] See Supplemental Material at http://link.aps.org/supple mental/10.1103/PhysRevApplied.11.044012 for additional data for experiments and solar cell fabrication and characterization details.

[38] S. B. Srivastava, S. K. Srivastava, and S. P. Singh, Molecular-shape-induced efficiency enhancement in PC61BM and PC71BM based ternary blend organic solar cells, J. Phys. Chem. C 121, 17104 (2017).

[39] L. Xu, Y.-J. Lee, and J. W. P. Hsu, Charge collection in bulk heterojunction organic photovoltaic devices: An impedance spectroscopy study, Appl. Phys. Lett. 105, 123904 (2014).

[40] G. Garcia-Belmonte, A. Munar, E. M. Barea, J. Bisquert, I. Ugarte, and R. Pacios, Charge carrier mobility and lifetime of organic bulk heterojunctions analyzed by impedance spectroscopy, Org. Electron. 9, 847 (2008).

[41] D. Rand, M. Jakesova, G. Lubin, I. Vebraite, M. DavidPur, V. Derek, T. Cramer, N. S. Sariciftci, Y. Hanein, and E. D. Glowacki, Direct electrical neurostimulation with organic pigment photocapacitors, Adv. Mater. 30, e1707292 (2018).

[42] C. H. Chuang, P. R. Brown, V. Bulovic, and M. G. Bawendi, Improved performance and stability in quantum dot solar cells through band alignment engineering, Nat. Mater. 13, 796 (2014).

[43] T. Ameri, P. Khoram, J. Min, and C. J. Brabec, Organic ternary solar cells: A review, Adv. Mater. 25, 4245 (2013).

[44] D. Shao, L. Zheng, D. Feng, J. He, R. Zhang, H. Liu, X. Zhang, Z. Lu, W. Wang, and W. Wang, TiO ${ }_{2}-\mathrm{P} 3 \mathrm{HT}$ : PCBM photoelectrochemical tandem cells for solar-driven overall water splitting, J. Mater. Chem. A 6, 4032 (2018).
[45] S. B. Srivastava, M. H. Modi, S. K. Ghosh, and S. P. Singh, Investigation of the buried planar interfaces in multilayered inverted organic solar cells using x-ray reflectivity and impedance spectroscopy, J. Phys. Condens. Matter 31, 124003 (2019).

[46] S. B. Srivastava, P. Sonar, and S. P. Singh, Analysis of degradation mechanisms in donor-acceptor copolymer based organic photovoltaic devices using impedance spectroscopy, Mater. Res. Expr. 3, 096202 (2016).

[47] S. B. Srivastava, P. Sonar, and S. P. Singh, Charge transport studies in donor-acceptor block copolymer PDPPTNT and PC71BM based inverted organic photovoltaic devices processed in room conditions, AIP Adv. 5, 077177 (2015).

[48] F. Wallrapp and P. Fromherz, $\mathrm{TiO}_{2}$ and $\mathrm{HfO}_{2}$ in electrolyteoxide-silicon configuration for applications in bioelectronics, J. Appl. Phys. 99, 114103 (2006).

[49] Y. Jiang, et al., Rational design of silicon structures for optically controlled multiscale biointerfaces, Nat. Biomed. Eng. 2, 508 (2018).

[50] M. Plaksin, E. Shapira, E. Kimmel, and S. Shoham, Thermal Transients Excite Neurons through Universal Intramembrane Mechanoelectrical Effects, Phys. Rev. X 8, 011043 (2018).

[51] A. Varghese, E. M. TenBroek, J. Coles Jr, and D. C. Sigg, Endogenous channels in HEK cells and potential roles in HCN ionic current measurements, Prog. Biophys. Mol. Biol. 90, 26 (2006).

[52] R. Parameswaran, J. L. Carvalho-de-Souza, Y. Jiang, M. J. Burke, J. F. Zimmerman, K. Koehler, A. W. Phillips, J. Yi, E. J. Adams, and F. Bezanilla, Photoelectrochemical modulation of neuronal activity with free-standing coaxial silicon nanowires, Nat. Nanotechnol. 13, 260 (2018).

[53] H. Bahmani Jalali, M. M. Aria, U. M. Dikbas, S. Sadeghi, B. G. Kumar, M. Sahin, I. H. Kavakli, C. W. Ow-Yang, and S. Nizamoglu, Effective neural photostimulation using indium-based type-II quantum dots, ACS Nano 12, 8104 (2018).

Correction: The surname of the eighth author contained a typographical error and has been fixed. A reference and its citation in Appendix B 2 were missing and have been inserted. 